\title{
Determining the WIMP mass from a single direct detection experiment $\left(15^{\prime}+5^{\prime}\right)$
}

\section{Anne Green*}

University of Nottingham

E-mail: anne.green@nottingham.ac.uk

\begin{abstract}
The energy spectrum of nuclear recoils in Weakly Interacting Massive Particle (WIMP) direct detection experiments depends on the underlying WIMP mass (strongly for light WIMPs, weakly for heavy WIMPs). We discuss how the accuracy with which the WIMP mass could be determined by a single direct detection experiment depends on the detector configuration and the WIMP properties. In particular we examine the effects of varying the underlying WIMP mass, the detector target nucleus, exposure, energy threshold and maximum energy, the local velocity distribution and the background event rate and spectrum.
\end{abstract}

Identification of dark matter 2008

August 18-22, 2008

Stockholm, Sweden

\footnotetext{
* Speaker.
} 


\section{Introduction}

The direct detection of WIMPs in the lab would not only directly confirm the existence of dark matter but would also allow us to probe the WIMP properties, in particular its mass. This would shed light on its nature and probe extensions of the standard model of particle physics. Furthermore definitive detection of the WIMP may well require consistent signals (i.e. with the same inferred WIMP properties) from direct detection, indirect detection and collider experiments. Here we give a brief overview of recent work [1] on determining the WIMP mass from direct detection experiments (see also Refs. [目, 纤, 团).

The differential event rate (number of events per unit energy, time and detector mass) has a roughly exponential energy dependence: [记]

$$
\frac{\mathrm{d} R}{\mathrm{~d} E} \approx c_{1} F^{2}(E)\left(\frac{\mathrm{d} R}{\mathrm{~d} E}\right)_{0} \exp \left(-\frac{E}{c_{2} E_{\mathrm{R}}}\right)
$$

where $c_{1}$ and $c_{2}$ are fitting parameters of order unity (which depend on the target mass number and energy threshold), $(\mathrm{d} R / \mathrm{d} E)_{0}$ is the event rate in the $E \rightarrow 0 \mathrm{keV}$ limit and $F(E)$ is the form factor. The characteristic energy scale of the exponential, $E_{\mathrm{R}}$, depends on the WIMP mass, $m_{\chi}$, and is given by

$$
E_{\mathrm{R}}=\frac{2 m_{A} m_{\chi}^{2} v_{\mathrm{c}}^{2}}{\left(m_{\chi}+m_{A}\right)^{2}}
$$

where $m_{A}$ is the target nuclei mass and $v_{\mathrm{c}}$ is the local circular speed. For light WIMPs $\left(m_{\chi} \ll m_{\mathrm{A}}\right)$ $E_{\mathrm{R}} \propto m_{\chi}^{2}$, while for heavy WIMPs $\left(m_{\chi} \gg m_{\mathrm{A}}\right) E_{\mathrm{R}} \sim$ const. In other words, for light WIMPs the energy spectrum is strongly dependent on the WIMP mass while for heavy WIMPs the dependence on the WIMP mass is far weaker. Consequently it should be easier to measure the mass of light (compared with the target nuclei) WIMPs than heavy WIMPs.

\section{Monte Carlo simulations}

We have used Monte Carlo simulations to examine how well a SuperCDMS like detector [5] could determine the WIMP mass from the energies of observed WIMP nuclear recoil events. Our benchmark detector is composed of $\mathrm{Ge}$, has a nuclear recoil energy threshold of $E_{\text {th }}=10 \mathrm{keV}$ and has no upper limit on the recoil energy. We assume that the detection efficiency is independent of energy, the energy resolution is perfect, the background is zero, the form factor has the Helm form and fix the WIMP-proton cross-section to be $\sigma_{\mathrm{p}}=10^{-8} \mathrm{pb}$, a factor of a few below the current exclusion limits [6]. We assume the local WIMP speed distribution is Maxwellian and the local density is $0.3 \mathrm{GeV} \mathrm{cm}^{-3}$ and consider (efficiency weighted) exposures of $\mathscr{E}=3 \times 10^{3}, 3 \times 10^{4}$ and $3 \times 10^{5} \mathrm{~kg}$ day which correspond, roughly, to a detector with mass equal to that of the 3 proposed phases of SuperCDMS taking data for a year with a $\sim 50 \%$ detection efficiency. These are, generally, optimistic assumptions and will therefore give 'best case' results. We discuss the effects of dropping or varying some of these assumptions in Sec. 3, see also Ref. [1].

For each WIMP mass, $m_{\chi}^{\text {in }}$, and detector configuration we calculate the probability distribution of the maximum likelihood estimator of the WIMP mass by simulating $10^{4}$ experiments. We first calculate the expected number of events, $\lambda$, from the input energy spectrum. The actual number of 


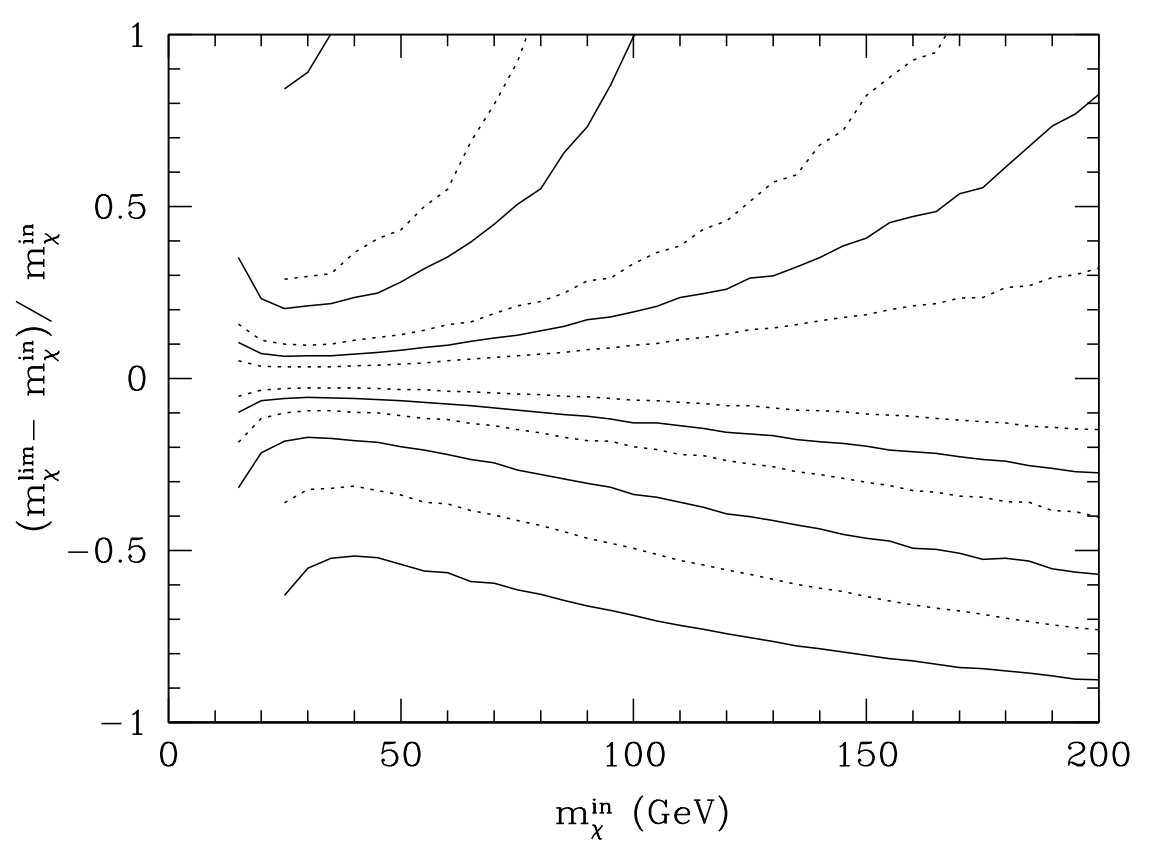

Figure 1: The fractional deviation of the WIMP mass limits from the input mass, $\left(m_{\chi}^{\lim }-m_{\chi}^{\text {in }}\right) / m_{\chi}^{\text {in }}$, for exposures $\mathscr{E}=3 \times 10^{3}, 3 \times 10^{4}$ and $3 \times 10^{5} \mathrm{~kg}$ day and input cross-section $\sigma_{\mathrm{p}}=10^{-8} \mathrm{pb}$ for the benchmark SuperCDMS like detector. The solid (dotted) lines are the $95 \%$ (68\%) confidence limits.

events for a given experiment, $N_{\text {expt }}$, is drawn from a Poisson distribution with mean $\lambda$. We Monte Carlo generate $N_{\text {expt }}$ events from the input energy spectrum, from which the maximum likelihood mass and cross-section for that experiment are calculated. Finally we find the (two-sided) 68\% and 95\% confidence limits on the WIMP mass from the maximum likelihood masses.

\section{Results and discussion}

The accuracy with which the WIMP mass could be measured by the benchmark SuperCDMS [5] like Ge detector described above is shown in Fig. 1. With exposures of $\mathscr{E}=3 \times 10^{4}$ and $3 \times$ $10^{5} \mathrm{~kg}$ day it would be possible to measure the mass of a light, $m_{\chi} \sim \mathscr{O}(50 \mathrm{GeV})$, WIMP with an accuracy of roughly $25 \%$ and $10 \%$ respectively. For heavy WIMPs $\left(m_{\chi} \gg 100 \mathrm{GeV}\right)$ even with a large exposure it will only be possible to place a lower limit on the mass. For very light WIMPs, $m_{\chi}<\mathscr{O}(20 \mathrm{GeV})$, the number of events above the detector energy threshold would be too small to allow the mass to be measured accurately.

The number of events detected is directly proportional to both the exposure and the crosssection, therefore these quantities have the greatest bearing on the accuracy of the WIMP mass determination.

The energy threshold, $E_{\mathrm{th}}$, and the maximum energy, $E_{\max }$, above which recoils are not detected/analysed also affect the accuracy with which the WIMP mass can be determined. Increasing $E_{\text {th }}$ (or decreasing $E_{\max }$ ) not only reduces the number of events detected, but also reduces the range of recoil energies and the accuracy with which the characteristic energy of the energy spectrum, 
$E_{\mathrm{R}}$, and hence the WIMP mass, can be measured. For light WIMPs the small $E_{\mathrm{R}}$ means that the expected number of events decreases rapidly as the energy threshold is increased, while for heavy WIMPs the large $E_{\mathrm{R}}$, and flatter energy spectrum, means that the smaller range of recoil energies reduces the accuracy with which $E_{\mathrm{R}}$ can be measured. Reducing the maximum energy only has a significant effect for heavy WIMPs.

The WIMP and target mass dependence of $E_{\mathrm{R}}$ suggests that heavy targets will be able to measure the mass of a heavy WIMP more accurately, however the rapid decrease of the nuclear form factor with increasing momentum transfer which occurs for heavy nuclei means that this is in fact not the case (see also Ref. [四).

If the WIMP distribution on the ultra-local scales probed by direct detection experiments is smooth, then the $\pm 20 \mathrm{~km} \mathrm{~s}^{-1}$ uncertainty in the local circular speed [7] leads to a $\sim 10 \%$ systematic error in the determination of $m_{\chi}$. Changes in the detailed shape of the local velocity distribution lead to relatively small changes in the shape of the differential event rate [8], and hence a relatively small, $\mathscr{O}(5 \%)$, systematic uncertainty in the WIMP mass. If the ultra-local WIMP distribution consists of a finite number of streams, then the energy spectrum will consists of a number of steps. The positions of the steps will depend on the (unknown) stream velocities, as well as the target nuclei and WIMP masses. With multiple targets it would in principle be possible to constrain the WIMP mass without making any assumptions about the WIMP velocity distribution [4].

Future experiments aim to have negligible backgrounds, however if the background rate is not negligible compared with the WIMP event rate it will be difficult to disentangle a WIMP signal (and the WIMP mass) from the background if the background spectrum has a similar shape to the WIMP spectrum (i.e. exponential background, or flat background and a heavy WIMP). The uncertainties from backgrounds could be mitigated by using multiple targets and/or using multiple scatter events to measure/constrain the background spectrum.

\section{References}

[1] A. M. Green, JCAP08(2007)022, hep-ph/0703217; JCAP07(2008)005, arXiv:0 005.1704.

[2] J. D. Lewin and P. F. Smith, Astropart. Phys. 6, 87 (1996).

[3] M. J. Lewis and K. Freese, Phys. Rev. D 70043501 (2004), astro-ph/ 0307190 ; J. L. Bourjaily and G. L. Kane, hep-ph/0501262; D. Jackson, R. Gaitskell and R. W. Schnee, unpublished (2006).

[4] M. Drees and C-L. Shan, JCAP06(2008)012, arXiv: 0803.4477.

[5] R. W. Schnee et al., astro-ph/0502435; P. L. Brink et al., astro-ph/0503583.

[6] J. Angle et al., Phys. Rev. Lett. 100, 021303 (2008), arXiv: 0706.0039 , Z. Ahmed et al., arXiv: 0802.3530 (2008).

[7] F. J. Kerr and D. Lynden-Bell, Mon. Not. Roy. Astron. Soc. 221, 1023 (1986).

[8] M. Kamionkowski and A. Kinkhabwala, Phys. Rev. D 57, 3256 (1998), hep-ph / 9710337 ; F. Donato, N. Fornengo and S. Scopel, Astropart. Phys. 9, 247 (1998), hep-ph/ 9803295. 